\title{
CLINICAL, HEMATOLOGICAL AND PARASITOLOGICAL STUDY ON CANINE BABESIOSIS
}

\author{
Ali A Abdel-Rahman ', Yamen, M. Hegazy', Khaled Sultan', \\ Magdy H. Al-Gaabary ${ }^{l}$ \\ ${ }^{1}$ Animal Medicine Depart., Fac. of Vet. Med., Kafrelsheikh Univ., \\ 33516 Kafrelsheikh, Egypt. \\ ${ }^{2}$ Parasitology Depart., Fac. of Vet. Med., Kafrelsheikh Univ., \\ 33516 Kafrelsheikh, Egypt.
}

\begin{abstract}
Canine babesiosis is a common tick-borne disease of dogs worldwide. A number of Babesia sp. can infect dogs and the spectrum is increasing as molecular methods are developed to differentiate organisms. In the current study between March, 2014 and February, 2015, a total number of 200 dogs were examined for babesiosis. The clinical findings, the vector tick identification, hematological changes and parasitological diagnosis (blood film examination) of Babesia were investigated. Animals in cute form showed animals in this form showed fever (39.3 - 41.5 C), lethargy, icterus, splenomegaly, vomiting, congested mucous membrane, and hematuria. While chronic form characterized by intermittent pyrexia, partial anorexia, loss of body condition, pale mucous membrane. On the other hand, subclinical form shows no symptoms. Results of blood film showed that Babesia canis trophozoite existed in 2 morphological phenotypes either as piriform type or as an amoeboid type. Out of 200 dogs examined; 79 (39.5\%) were naturally infected with hard ticks. The hard tick species were identified as Rhipicephalus sanguineus. The hematological findings showed that, there was significant decrease in RBCs count, Platelets count, Granulocytes count, HCT, and HGB in animals of infected group than healthy animals.
\end{abstract}




\section{INTRODUCTION}

Babesiosis is a malaria-like, zoonotic disease transmitted by ticks and caused by protozoa of the genus Babesia, which invade and destroy erythrocytes. (George et al., 2011).

Canine babesiosis is an infection of great veterinary importance worldwide. The disease is characterized by hemolytic anemia, fever, and splenomegaly. Babesia infections can also be subclinical or cause severe life-threatening .The major clinical signs are illness, Lethargy, pallor, splenomegaly (Birkenheuer, 2014).

There is a very strong association between the Babesia species that is transmitted and the tick species. As a consequence, the prevalence of babesiosis is dependent on the presence of the tick vector in the environment (Matijatko et al., 2012).

B. vogeli is transmitted by $R$. sanguineus causing mild disease in dogs. Presence of infected dogs is not required for $B$. vogeli to remain in the tick population (Taboada and Merchant, 1991).

Rhipicephalus sanguineus, the brown dog tick, is widely distributed on dogs across the world; the tick is well adapted for tropical climates and is associated with year round infestations (Dantas-Torres, 2010).

There are limited studies on canine babesiosis in Egypt and most of studies and veterinarians consider it is Ehrlichiosis. So, the aim of this study is to investigate the clinical and hematological abnormalities associated with canine babesiosis and to study the role of ticks for its transmission. 


\section{MATERIAL AND METHODS}

\section{1- Animals and physical examination:}

Between March, 2014 and February, 2015, a total number of 200 dogs were examined with great consideration to animal welfare. Dogs were belonged to the Training Center for security services in Alexandria (K9), Training Center for security services in EL-Abasya (K9), Cairo, Dog shelter at 6 October City, Giza, Dog shelter at EL-Obour, Qaluobia, and cases presented to private pet clinic and Faculty of Veterinary Medicine, educational clinics, Kaferelsheikh University. Each animal was subjected to complete clinical examination including: general inspection of appearance, posture, gait, illness, conformation, skin, behavior, Physical examination for temperature, respiration, pulse, mucous membranes and superficial lymph nodes (Hill et al., 2011).

\section{2- Samples and parasitological examination:}

\section{- Blood smear:}

Blood smears were obtained from capillaries; tip of ear for detection of hemoparasite stages under the microscope. Blood smears were prepared, the smears were air dried, fixed in methanol for three minute and stained in $10 \%$ Giemsa stain for $20-30 \mathrm{~min}$. then washed with distilled water, air dried and examined microscopically under oil immersion lens (X1000) for detection of Babesia parasite inside erythrocytes.

\section{- Tick samples:}

Dogs were screened for presence of hard ticks by the aid of hand lenses and a fine tooth-comb. Samples were collected by careful removing of ticks by hand, counted, fixed in $70 \%$ ethanol. For 
identification, some specimens were cleared in polyvenyl lactophenol and mounted as described by (Price \& Graham, 1997). The collected ticks were identified according to (Solusby, 1982).

\section{3- Samples and hematological examination:}

EDTA-whole blood samples (4 $\mathrm{ml}$ each) were collected from each dog from the cephalic vein (fore limb) or saphenous vein (hind limb) and used for hematological studies (C.B.C). Hematological analysis was done using veterinary blood cells counter (Medonic Ca, 620, Sweden) which is a single analyzer system, using the same electronic particle counting technology and the same software for calculating and reporting results.

The statistical differences between infected animals and healthy animals in blood parameters were estimated using ANOVA on SPSS 21.

\section{RESULTS}

\section{Clinical findings:}

- There are signs which occurred in infected cases such as anorexia, lethargy, weakness, pyrexia, weight loss, diarrhea, constipation, tick infestation, congested mucous membrane, ocular and nasal discharge, respiratory distress, back pain.

- All of infected puppies were died (3 puppies) even after administration of medication and on the other hand there was no mortalities in adult infected dogs.

\section{Results of parasitological examination:}

- Results of blood film showed that Babesia canis trophozoite existed in 2 morphological phenotypes either as piriform type or as an amoeboid type figure a: 5 . 
Table (1): Prevalence rate of canine babesiosis in examined dogs by blood film.

\begin{tabular}{|c|c||c|}
\hline Total No. of examined dogs & No. of positive cases & Prevalence rate \\
\hline \hline 200 & 17 & $8.5 \%$ \\
\hline
\end{tabular}

- Out of 200 dogs examined; 79 (39.5\%) were naturally infected with hard ticks figures A: 3 and 4. The hard tick species were identified as Rhipicephalus sanguineus (Arachnida: Acari: Ixodidae) figure A: 6; it is also known as brown dog tick. Briefly, it is red-brown in coloration, lacking any distinctive markings or body patterns with an elongated body shape and hexagonally shaped mouth parts; the later morphological feature is characteristic for this species.

Table (2): Prevalence rate of tick infestation in examined dogs.

\begin{tabular}{|c||c|c||}
\hline \hline Total No. of examined dogs & No. of dog have tick infestation & Prevalence rate \\
\hline \hline 200 & 79 & $39.5 \%$ \\
\hline
\end{tabular}

\section{Results of hematological examination:}

The examined animals were divided into two groups:

Group (A) Consists of animals proved to be naturally infected with parasite.

Group (B) Consists of animals free of parasite.

The mean values of blood parameters for animals of the 2 groups are showed in table 1.The statistical analysis of the difference in these parameters among animals of both groups is showed in table 2.These findings showed that there was significant decrease in RBCs count, Platelets count, Granulocytes count, HCT, and HGB in animals of infected group than healthy animals. On the other hand the decrement in WBCs count, MID count and MPV count in infected animals were not significant. There was an increment in Lymphocytes count in infected animals than normal animals but this finding was not significant. 
Ali A Abdel-Rahman et., al.
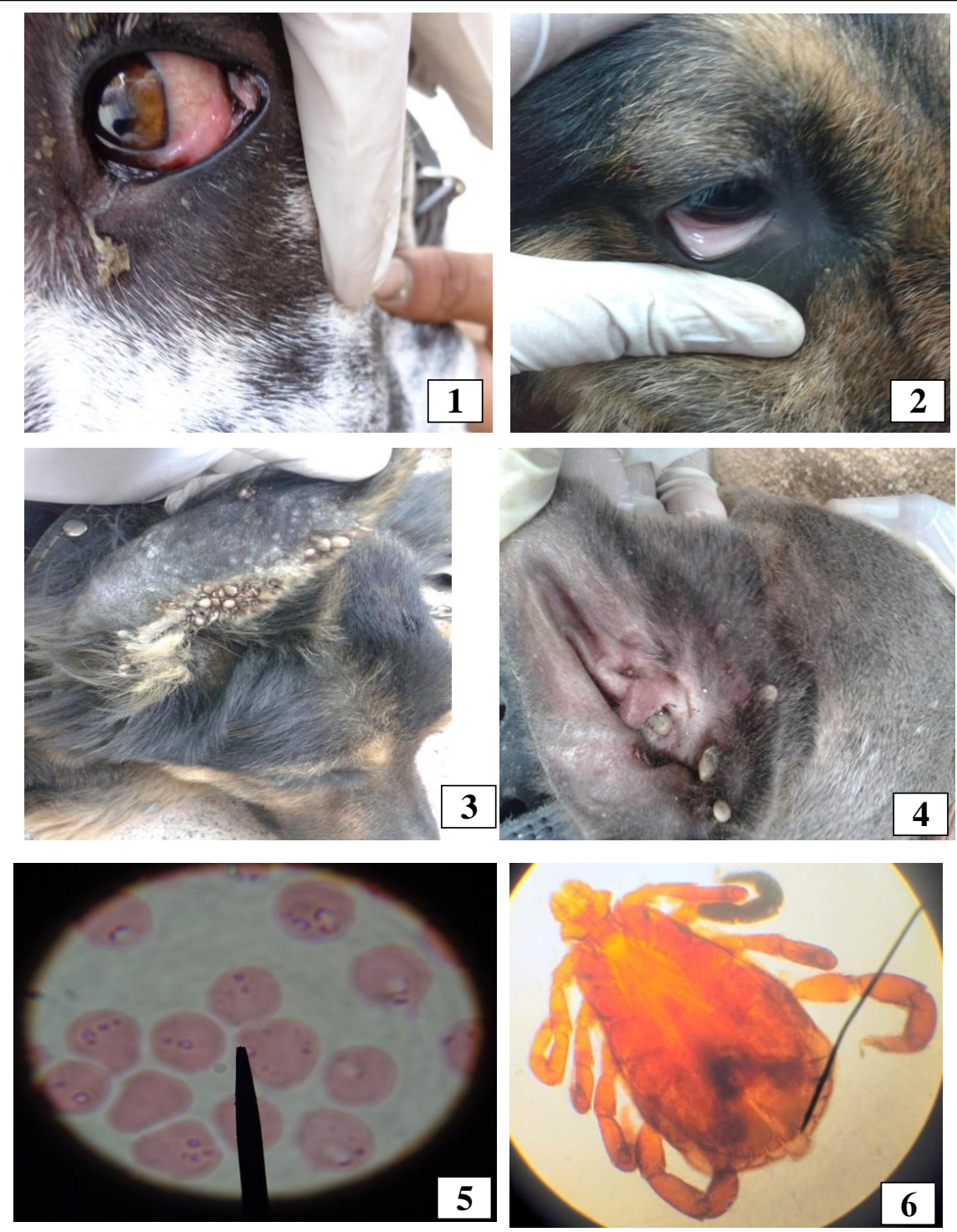

Fig. (A): 1: Engaged eye capillaries and congested mucous membranes in male Great Dane dog. 2: Pale mucous membranes in male German shepherd dog. 3: tick infestation in ear of German shepherd 4: Tick infestation in dogs in the inner aspect of the ear in male pit bull. 5: Blood smear showing different stages of trophozoites babesia canis. 6: Male Rhipicephalus sanguineus sensu lato. Note the hexagonal basic capitulumin the anterior end and 9 festoons in the posterior margin. 
Clinical, Hematological And Parasitological Study On ...

Table (3): Mean values of blood parameters of infected and control (None infected animals).

\begin{tabular}{|c|c|c|}
\hline Parameters & infected with parasite & Healthy non infected (control) \\
\hline $\operatorname{RBCs}\left(10^{6} / \mathrm{cmm}\right)$ & 5.77 & 6.71 \\
\hline MCV um 3 & 62.15 & 62.05 \\
\hline RDW\% & 17.03 & 17.38 \\
\hline RDWA um 3 & 44.92 & 45.29 \\
\hline НCT \% & 35.82 & 41.74 \\
\hline PLT $103 / \mathrm{mm} 3$ & 263.82 & 310.68 \\
\hline MPV um 3 & 7.68 & 16.27 \\
\hline PDW um 3 & 10.88 & 10.77 \\
\hline PCT $\%$ & 0.20 & 0.47 \\
\hline LPCR \% & 16.96 & 16.64 \\
\hline WBC $10 \quad 3 / \mathrm{mm} 3^{2}$ & 14.49 & 15.57 \\
\hline HGB g/dl & 13.42 & 15.585 \\
\hline MCH pg & 23.28 & 23.22 \\
\hline MCHC g/dl & 37.49 & 37.35 \\
\hline LYMF $103 / \mathrm{mm} 3$ & 7.28 & 6.76 \\
\hline GRAN $103 / \mathrm{mm} 3$ & 4.45 & 5.88 \\
\hline MID $10 \quad 3 / \mathrm{mm} 3$ & 2.76 & 2.98 \\
\hline
\end{tabular}

Red blood cells count (RBCs), hemoglobin concentration (HGB), red blood cells distribution width (RDW), red blood cells distribution width absolute (RDWa), hematocrit (HCT), main corpuscular volume (MCV), mean corpuscular hemoglobin (MCH), mean corpuscular hemoglobin concentration (MCHC), platelets count (PLT), mean platelets volume (MPV), platelets distribution width (PDW), large platelets concentration ratio (LPCR), plateletcrit (PCT), white blood cells count (WBCs), Lymphocytes total count ( LYMF), Granulocytes total count (GRAN), Mid Cells total count (MID).

Table (4): Results of ANOVA for the significant differences between CBC parameters in healthy and infected dogs.

\begin{tabular}{|c|c|c|c|c|c|c|}
\hline \multicolumn{7}{|c|}{ ANOVA } \\
\hline & & Sum of Squares & Df & Mean Square & $\mathbf{F}$ & Sig. \\
\hline RDW & Between Groups & 1.648 & 1 & 1.648 & 1.628 & .205 \\
\hline RBCs & Between Groups & 12.564 & 1 & 12.564 & 34.850 & .000 \\
\hline $\mathrm{MCV}$ & Between Groups & .127 & 1 & .127 & .017 & .898 \\
\hline RDWA & Between Groups & 1.937 & 1 & 1.937 & .357 & .552 \\
\hline PLT & Between Groups & 30848.120 & 1 & 30848.120 & 4.481 & .037 \\
\hline MPV & Between Groups & 1035.426 & 1 & 1035.426 & .207 & .650 \\
\hline HCT & Between Groups & 493.569 & 1 & 493.569 & 31.704 & .000 \\
\hline PDW & Between Groups & .169 & 1 & .169 & .173 & .679 \\
\hline PCT & Between Groups & 1.001 & 1 & 1.001 & .276 & .600 \\
\hline LPCR & Between Groups & 1.463 & 1 & 1.463 & .060 & .807 \\
\hline WBC & Between Groups & 16.379 & 1 & 16.379 & .600 & .440 \\
\hline HGB & Between Groups & 65.657 & 1 & 65.657 & 31.223 & .000 \\
\hline $\mathrm{MCH}$ & Between Groups & .051 & 1 & .051 & .063 & .802 \\
\hline $\mathrm{MCHC}$ & Between Groups & .286 & 1 & .286 & .774 & .381 \\
\hline LYMF & Between Groups & 3.809 & 1 & 3.809 & .518 & .473 \\
\hline GRAN & Between Groups & 28.861 & 1 & 28.861 & 4.890 & .029 \\
\hline
\end{tabular}

Kafrelsheikh Vet. Med. J. Vol. 13 No. 2 (2015) 


\section{DISCUSSION}

In Egypt, there is an increase interest in dog breeding for different reasons: the first is dogs for the military serveries and Police, second, is dogs for special security services and third is the recent huge interest of using them as pet animals. This is besides the stray dogs in villages and towns. In spite of this noticed increase in numbers of dogs for different purposes, canines did not receive much attention from scientists as compared to other animals (Salem and Farag, 2014). This increase in dog population in Egypt may be responsible for transmitting some infectious diseases to humans or farm animals. Also, dog diseases may be responsible for hug monetary loss due to deaths occurred among imported dogs which are very expensive. The most significant infectious diseases affect dogs in Egypt are ehrlichiosis and babesiosis (Salem and Farag, 2014).

In this study, clinical signs were expressed in 3 forms: acute, chronic and subclinical. The acute form is mainly found in young puppies or adult dogs with weak immunity. Animals in this form showed high fever, red urine in puppies or dark yellow colored urine in adults, oculo-nasal discharge and enlarged lymph nodes, congested mucous membranes. The acute signs are not the common. The reason for this attributed to the low number of examined puppies and also due to endimicity of babesiosis in our dog population (Harvey et al., 1988). The chronic form and subclinical form are the main forms found in this study. These 2 forms are characterized by emaciated dogs, pale mucous membrane in chronic form and normal animals except a weak fever and off food in subclinical form. These 2 forms are the predominant forms due to the immunity of adult dogs, the weakness of the causative agent

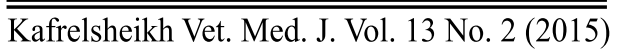


(Caccio et al., 2002; Irwin 2009, Ionita et al., 2012) as Babesia Vogeli is the weakest type of Babesia. Another reason for this finding is the periodic routine administration of Imidocarb by most of owners to their dogs which lead to some sort of partial prevention and this is the main reason for endemic stability.

Rhipicephalus sanguineus was the only tick detected in this study; this type of ticks is 3 host ticks and well-tailored to rural areas and it thrives as the biological vector of canine babesiosis. (Salem and Farag, 2014).

The hematological parameters showed that infected dogs suffered from significant anemia and this finding agrees with another finding of other authors (Gallego et al., 2008; Birkenheuer, 2014) and disagrees with others (Bourdoiseau, 2006). This may be attributed to the heterogeneity of the study populations and the variable sampling times. But most of time the anemia produced by babesia Vogeli is regenerative. (Scheepers et al., 2011).

The main hematological findings were thrombocytopenia, leucopenia and this agree with the findings of most past scientific articles (Gallego et al., 2008; Schetters, 2009; Zamokas et al., 2014).

Inspite of our findings, most of previous studies confirmed that Babesia vogeli has not homogenous clinico-pathological pattern and depends on the animal immunity, infection dose, and age....etc. (Gallego et al., 2008).

Finally, we concluded that further studies on canine infectious disease is much required to meet the huge increase in its number in Egypt recently to prevent the economic losses from their infection or either human or farm animals infections. 


\section{REFERENCES}

- Birkenheuer, A.J., 2014. Chapter 75, Babesiosis . Canine and Feline Infectious Diseases, St Louis: Elsevier; Pages 727-738.

- Bourdoiseau, G., 2006. Canine babesiosis in France. Vet. Parasitol. $138,118-125$.

- Caccio, S.M., Antunovic, B., Moretti, A., Mangili, V., Marinculic, A., Baric, R.R., Slemenda, S.B., Pieniazek, N.J., 2002. Molecular characterization of Babesia canis canis and Babesia canis vogeli from naturally infected European dogs. Vet. Parasitol. 106, 285-292.

- Dantas-Torres, F., 2010. Biology and ecology of the brown dog tick, Rhipicephalus sanguineus. Parasit. Vectors 3, 26, http://dx.doi.org/10. 1186/1756-3305-3-26.

- George, D. I., Ronald C. N., Francis M. C., 2011. Chapter 11, Babesiosis. From e-book, Topics on the Pathology of Protozoan and Invasive Arthropod Diseases. Armed Forces Institute of Pathology, Washington, DC, 20306

- Harvey, J.W., Taboada, J., Lewis. J.C., 1988. Babesiosis in a litter of pups. J. Am. Vet. Med. Assoc. 192, 1751-1752.

- Hill, P. B., Warman, S., Shawcross, G., 2011. One hundred top consultations in small animal general practice. ISBN 978-1-40516949-3.

- Ionita. M. ,. Mitrea. I. L., Buzatu. C. M., Pfister. K., Hamel. D., Silaghi. C., 2012. Canine babesiosis in Romania due to Babesia canis and Babesia vogeli: a molecular approach . Parasitol Res (2012) 110:1659-1664 
- Irwin, P.J., 2009. Canine babesiosis: from molecular taxonomy to control. Parasit. Vectors 2 (Suppl. 1), S4.

- Matijatko, V., Torti, M., Schetters ,T .P., 2012. Canine babesiosis in Europe: how many diseases? .Trends in Parasitology, Vol. 28, No. 3.

- Price, M., Graham, O., 1997. Chewing and Sucking Lice as Parasites of Mammals and Birds. USDA Agricultural Research Service Technical Bulletin 1849: 7-11.

- Salem, N. Y., Farag H. S., 2014. Clinical, Hematologic, and Molecular Findings in Naturally Occurring Babesia canis vogeli in Egyptian Dogs, Hindawi Publishing Corporation ,Veterinary Medicine International, Volume 2014, Article ID 270345, 6 pages .

- Scheepers E, Leisewitz AL, Thompson PN, Christopher MM. Serial haematology results in transfused and non-transfused dogs naturally infected with Babesia rossi. J S Afr Vet Assoc. 2011; 82(3):136-143.

- Schetters, T.P.M, Kleuskens, J.A.G.M, Van De Crommert, J., De Leeuw , P.W.J, Finizio , A.-L, Gorenflot , A. , 2009 . Systemic inflammatory responses in dogs experimentally infected with Babesia canis; a haematological study Veterinary Parasitology 162 ,7-15.

- Slousby E.J.L., 1982. Helminths, arthropods and protozoa of domesticated animals (7th edition), London: Bailliere Tindall, 809 pp., illus. ISBN 0-7020-0820-6.

- Taboada, J., Merchant, S.R., 1991. Babesiosis of companion animals and man. Veterinary Clinics of North America: Small Animal Practice, 21, 103-123.

- Zamokas. G., Grigonis. A., Karvelienè. B., Daunoras. G., Babickaitè. L., Šapalienè. I., 2014. Importance of haematological changes in diagnosing canine babesiosis. Issn 1392-2130. veterinarija ir zootechnika (vet med zoot). t. 67 (89). 